\title{
Optical Biopsy and Optical Pathology: Affordable Health Care Under Low-Resource Settings
}

\author{
Swathi Rao ${ }^{1}$, Reena John ${ }^{1}$, Ajeetkumar Patil ${ }^{1}$, Unnikrishnan V. K. ${ }^{1}$, Sajan D. George ${ }^{2}$, \\ Keerthilatha M. Pai ${ }^{3}$, Ravikiran Ongole ${ }^{4}$, Tom Devasia ${ }^{5}$, V. B. Kartha ${ }^{1}$, and Santhosh Chidangi ${ }^{1 *}$ \\ ${ }^{1}$ Centre of Excellence for Biophotonics, Department of Atomic and Molecular Physics, Manipal Academy of Higher \\ Education, Manipal, Karnataka 576104, India \\ ${ }^{2}$ Centre for Applied Nanoscience, Department of Atomic and Molecular Physics, Manipal Academy of Higher \\ Education, Manipal, Karnataka 576104, India \\ ${ }^{3}$ Department of Oral Medicine and Radiology, Manipal College of Dental Sciences, Manipal, Manipal Academy of \\ Higher Education, Manipal, Karnataka 576104, India \\ ${ }^{4}$ Department of Oral Medicine and Radiology, Manipal College of Dental Sciences, Mangalore 575001, Manipal \\ Academy of Higher Education, Manipal, Karnataka 576104, India \\ 5 Department of Cardiology, Kasturba Medical College, Manipal Academy of Higher Education, Manipal, Karnataka \\ 576104, India \\ * e-mail: santhosh.cls@manipal.edu
}

\begin{abstract}
In this paper the potential of optical spectroscopy based techniques for regular screening and early diagnosis of "Killer" diseases (various types of cancers, cardiovascular diseases, etc.) is discussed with focus on routine use under low-resource settings. A brief account of work carried out in our laboratory to design, assemble and optimize Laser Induced Fluorescence (LIF) based optical devices for routine screening, early diagnosis and discrimination of premalignant/malignant conditions in oral and other cancers is presented. An ultra-sensitive protein profiling system based on highly efficient High Performance Liquid ChromatograpyUltrasensitive Laser Induced Fluorescence (HPLC-LIF) detection, and its extensive use in monitoring various cancers, coronary conditions, gynecological problems etc. through protein profile pattern analyses of markers in body fluids is also discussed. (C) 2020 Journal of Biomedical Photonics \& Engineering.
\end{abstract}

Keywords: optical pathology; laser induced fluorescence; HPLC-LIF; oral cancer; cardio vascular disease; principal component analysis.

Paper \#3377 received 12 Jun 2020; accepted for publication 17 Jun 2020; published online 30 Jun 2020. doi: 10.18287/JBPE20.06.020309.

\section{Introduction}

As of March 2020, the estimated world population stands at $>7.775$ billion [1]. The 32 OECD (Organization of Economic Development and Cooperation) "developed" countries all together constitute only $15 \%$ of this, of which $81 \%$ live in urban areas [2] with universal accessibility and availability of complete health care. In contrast, the remaining more than 6 billion people of the world, in developing countries, live with grossly inadequate health care facilities. This has lead, combined with the huge disparity in Per Capita Income (eg. US $>\$ 56,000$ vs
India $<\$ 6900)$ to a situation in which routine health care has become a commodity almost unavailable and unaffordable for the bulk of the population. Whatever modern facilities required for advanced health care are mostly located in big towns and large cities, making it mandatory for a rural person to take leave from his daily job in the village, stay away for several days to visit a large hospital in the city, incurring considerable expenditure for stay and for expensive medical tests. Often, for many disease conditions repeated visits become necessary, and many times the person has to have support of additional manpower, to facilitate his stay and therapy. The small hospitals and health care 
centers in rural areas have no facilities for regular screening for early detection of "Killer" diseases (coronary diseases, various types of cancer, tuberculosis, etc.), since such facilities (imaging techniques, detection of tumor markers, identification of drug-resistant pathogens, to mention a few) require costly equipment and experienced professionals (radiologists, pathologists) for routine operation, both of them unavailable in small rural hospitals. To cap all this, there is almost no awareness of the need for regular screening for major diseases for early detection and successful therapy. Finally, the rural population mostly consist of orthodox individuals, who are very reluctant to go for personally invasive diagnostic techniques like mammography, colposcopy, Trans-vaginal sonography, colonoscopy, etc., physical exposure to strangers, especially for women, being almost taboo. The total outcome of all these deficiencies is a humongous health care burden on the country in terms of manpower loss, economy, societal well-being, and human welfare index. In an analysis of global burden of disease study of 2015 , India is ranked as $143^{\text {rd }}$, out of 188 countries worldwide, in health related sustainable development goals [3]. It is clear that to escape from this deplorable social, financial, psychological, and human welfare conditions, one has to have universal health care, characterized by ready availability, affordability, and acceptability; that is, a cost-effective health surveillance program, which can be executed without the need for highly-qualified medical professionals. with minimum personal intrusion, and which can be made available at a large number of locations, like rural health-care centers, small hospitals, and single-doctor clinics in small towns and villages.

\section{Systems, Techniques, and Technology}

Optical pathology and optical biopsy are uniquely positioned at present, to provide highly cost effective, non- or minimally- invasive, point-of-use or field deliverable diagnostic methods. Optical methods can provide unambiguous, objective diagnosis in many biomedical applications like detection of pathogens in samples or environment, screening and early detection of asymptomatic abnormal health conditions, diagnosis and staging of diseases, prognosis and follow up in therapy, imaging applications, drug cell interactions, clinical trials, etc.

Furthermore, most of the time, they require only minimally trained technicians for routine operation, eliminating the need for highly qualified professionals. Revolutionary advances in lasers and other light sources like LEDs, miniaturization of spectroscopic instrumentation with mini and micro spectrometers, high sensitivity state-of-the-art detectors, and efficient and fast data processing techniques, have made optodiagnostic systems highly cost effective, amenable to large scale production of inexpensive portable, miniature, or wearable configurations, usable at almost any location.

All optical biomedical systems depend on the interaction of radiation with the material at the point of incidence [4]. The interaction involves three processes; absorption, reflection, or scattering of photons. Absorption can give rise to further processes, in which the absorbed energy is released either as a different photon (giving fluorescence) or as thermal energy, giving a photothermal effect. If the incident radiation is not continuous, but pulsed or periodically interrupted, the thermal excitation and relaxation will lead to acoustic waves which effect is called the photo acoustic effect. Since absorption will depend on the energy levels of the atoms/molecules in the system, all these processes will be highly characteristic of the structure and composition of the material under study.

Reflection of radiation from a material can be of two types. Conventional regular (specular, mirror-like, directional) reflection, obeying the laws of reflection, or diffuse reflection in which the radiation enters into the medium, gets multiply scattered, and exits in all directions (nondirectional). In the process of multiple scattering in the medium, some of the radiation can be absorbed, depending on the composition of the material, and hence the diffusely reflected radiation, once again, will be characteristic of the material, and can thus be used for getting information on its structure and composition. If, instead of the multiply scattered light in the reflection method, one uses singly scattered light, then one can apply interferometric methods to get information on the tissue structure. This is the basis of imaging by Optical Coherence Tomography (OCT) [5].

Scattering can be elastic (without loss or gain of energy for the incident the photon) or inelastic with some energy transfer between the incident photon and atoms/molecules in the medium. Elastic scattering can be approximated by Mie theory and has been developed for many imaging applications [6, 7]. The inelastic scattering with energy exchange - Raman scattering gives information on molecular characteristics, which can be used for many applications for in vivo, ex vivo, and in vitro diagnostic techniques [4].

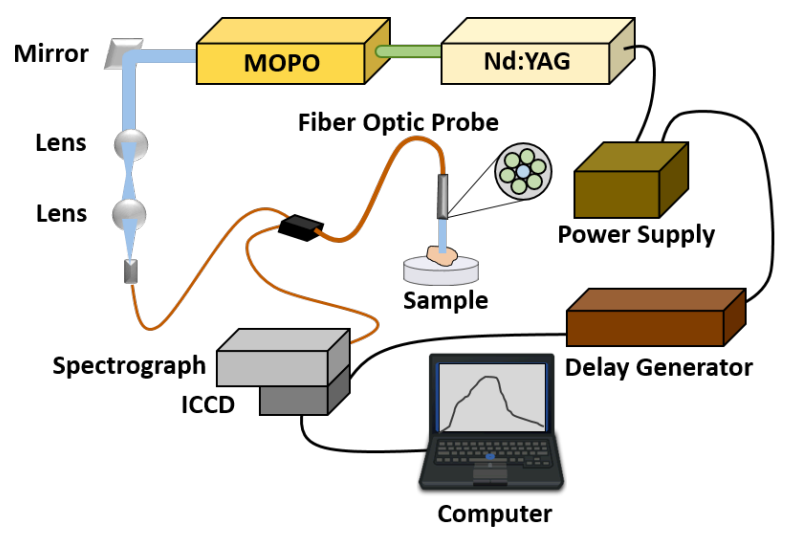

Fig. 1 Block diagram of the pulsed laser LIF setup.

In summary, for biomedical applications by optical techniques, the general instrumentation consists of an optical radiation source (laser, LED, lamp), a probe to direct the radiation to the sample and transmit the effect 
produced on it (by absorption, fluorescence, reflection, or scattering) to a radiation detector and analyze the return signal through various data processing techniques. Schematic diagram of such a system is shown in Fig. 1.

The system, in this specific case comprises of a pulsed Nd-YAG laser, a $150 \mathrm{~mm}$ spectrograph and an ICCD detector, can be used only in the lab because of its size, and operational requirements like power supply, Pure $\mathrm{N}_{2}$ gas for flushing, vibration isolation for optical components etc., because of these requirements it is not convenient for use in locations like small hospitals and clinics. We have used it extensively to develop ex vivo optical biopsy for various cancers, study of radiation and drug effects in radiotherapy and animal models, etc. [8-11].

A biopsy, by definition, "is a procedure to remove a piece of tissue or a sample of cells from your body so that it can be analyzed in a laboratory" [12]. Thus, conventional biopsy needs examination of the organ by a clinician or oncologist and removal of sample from visually suspect locations, depending on his judgment based on visual inspection. Often the process is highly subjective, may require removal of tissue samples from many locations, and prone to errors due to effects like "Field cancerization" [13], and mistakes due to "past pointing" etc., which can happen even with some experience. Also removal of tissue from suspect locations itself may lead to problems later. Further, the final decision as to the status of the sample is made by the pathologist, who again takes a decision based on visual examination and previous experience. With optical methods, an objective and unambiguous decision can be made by examination of multiple sites without the need for tissue removal and subsequent dangers. For such in vivo examinations the Nd-YAG-SpectrographICCD model is unsuitable because it is bulky and also fairly expensive. Both these drawbacks were eliminated in the next version of our portable optical biopsy system, by replacing the Nd-YAG laser with a much smaller, low power, and much cheaper continuous wave He-Cd laser, a less expensive CCD system and a fiber optic probe for delivery of the laser radiation and collection of the resulting spectra. This is shown in Fig. 2, clearly illustrating its ease of use for routine in vivo studies at any location.

The system was evaluated by in vivo examination of $\sim 380$ subjects (133 normal, 155 oral premalignancy, and 92 oral malignancy) and recording a total of about 4000 fluorescence spectra from different sites - buccal mucosa, tongue lateral, tongue tip, tongue top, tongue bottom, lip underside, and palate - under normal, premalignant, and malignant conditions [14]. The system in Fig. 2 is compact, portable, and can be operated by a technician. By putting different fiber optic probes, it can be easily adapted for use in all types of endoscopic examinations, like gastroscopy, colonoscopy, colposcopy etc. The diagnostic conclusions are arrived at by statistical pattern analysis methods like Principal Component Analysis (PCA) and artificial Neural
Network (ANN) [8-11], leading to highly objective decision making, with well defined statistical probability assigned to the decision [15]. Since no tissue removal is involved, a subject can be examined at as many locations as needed during any visit. Susceptible population groups (smokers, females, etc) can be called for regular periodic screening, since it is sufficiently cost effective for installation in small hospitals, clinics and community-health care centers.
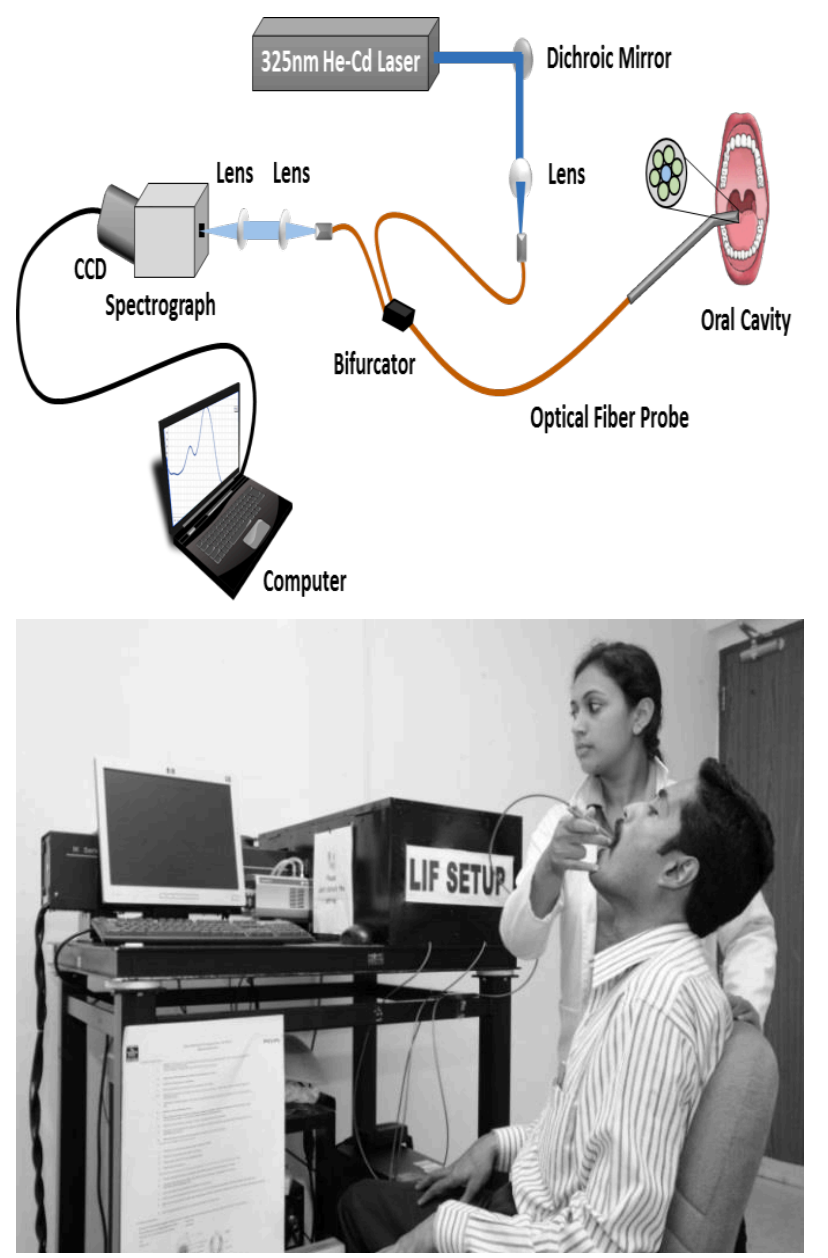

Fig. 2 Block diagram and the Photograph of the He-Cd laser based tabletop LIF system.

Even when facilities like that have shown in Fig. 2 become available in small town hospitals and clinics, regular health coverage by screening for the many types of cancers like oral, esophageal, cervical, etc. may not happen. The susceptible rural population, consisting of laborers, females busy with household chores, etc. usually pay very little attention to early symptoms of such diseases. Also they are often unaware of the need for such screening, and so neglect to have such examinations out of lack of leisure time or even sheer laziness. It is thus advantageous to have a system, which can be taken around to small health care camps, or which can be carried around by a health care worker for home visits. We achieved this easy translation ability by replacing the spectrograph-CCD combination with spectrograph-Photo Diode Array (PDA) unit, which can 
be incorporated in a hand-held unit. This is shown in Fig. 3a, b. Typical fluorescence spectra recorded from induced mice skin cancer using the setup are shown in Figure 3c. The He-Cd laser can be easily replaced with an UV light emitting LED with $325 \mathrm{~nm}$ sharp radiation to make the system still smaller and cheaper.

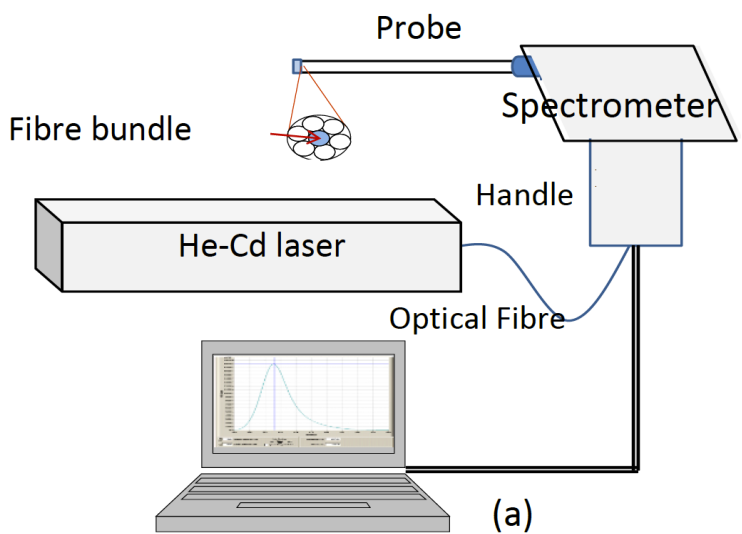

(a)

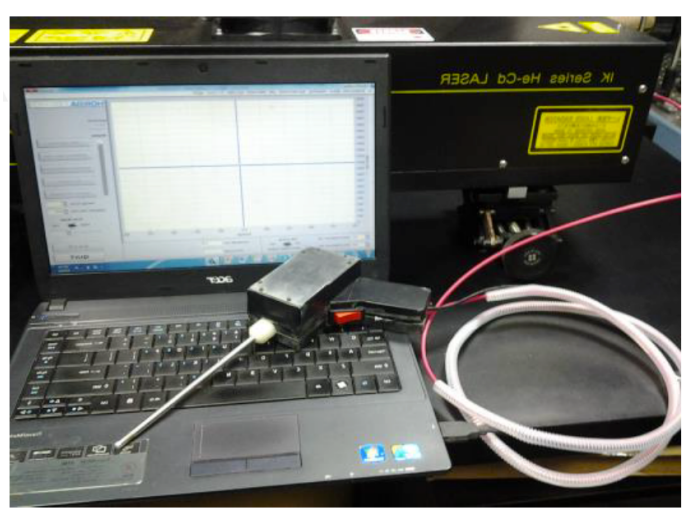

(b)

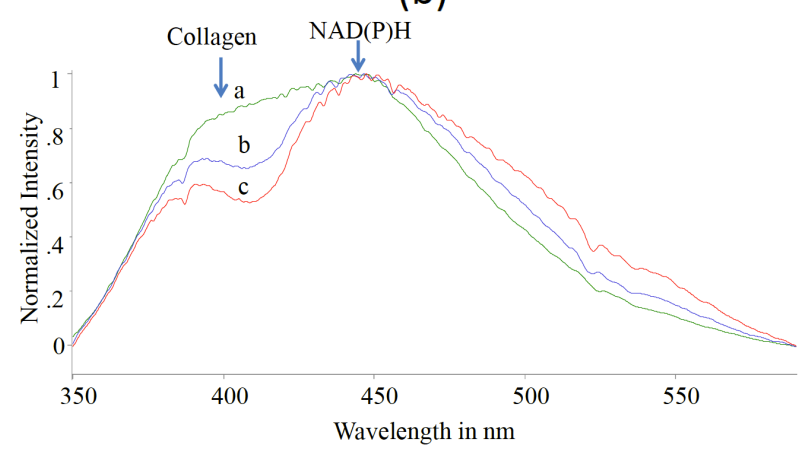

(c)

Fig. 3 Hand-held optical biopsy system for in vivo cancer screening. Typical autofluorescence spectra of healthy and cancer-induced mice skin recorded by using

the system; (a) Healthy, (b) Premalignant, and

(c) Malignant

The first requirement of the screening device was to show that its performance will be quite adequate for routine in vivo fluorescence spectroscopy. For this a detailed fluorescence spectroscopy study of squamous epithelial carcinogenesis in Swiss Albino mice was carried out. Swiss albino mice treated with 7,12-dimethylbenz(a)anthracene (DMBA) were used for this study. DMBA is a complete chemical cancirogen (initiator and promoter) and studies have shown that there is a consistent sequence of histological changes that transpire the DMBA treated epithelial layer of the mice skin lesion of Swiss Albino mice. The autofluorescence emission spectra at $325 \mathrm{~nm}$ excitation from DMBA treated animal skins and control animal skins were recorded (Fig. 3c) every week using the LIF system and the results were analyzed by statistical pattern analysis methods. Specificities and sensitivities, for discrimination between malignant and normal conditions have been calculated from the analysis and are found to be $89 \%$ and $91 \%$ respectively [16]. Showing that such a system is quite adequate to serve the purpose of screening and early diagnosis of cancers in organs accessible through endoscopic procedures.

It is very important to note that the same system, with minor modifications, can be adapted for other techniques of optodiagnostics applications, using Raman, Photo-acoustic, or Diffuse Reflection spectroscopy, in conditions like skin problems, arthritis, diabetes, etc.

\section{3 "Killer" Diseases}

According to a recent survey [17], the major "Killer" diseases in India (by percentage of total number of deaths) are cardiovascular diseases $(25 \%)$, respiratory diseases $(10 \%)$, Tuberculosis $(10 \%)$, cancer $(9 \%)$, digestive diseases $(5 \%)$, diarrhea $(5 \%)$, and malaria $(3 \%)$.

There are several reasons for these diseases to dominate the ill-health status of countries like India. The easily available screening facilities in developed countries lead to early detection in the majority urban population. But in developing countries, the majorityrural population is not diagnosed at all in the early stages. The easy availability of early detection methods to the majority urban population lead to a twice-higher detection of incidence rate, more successful therapy, and consequent higher survival rate in developed countries, but leads to more or less similar death rates in both cases [18]. Currently, there are no screening methods routinely applied for the $70 \%$ rural population in countries like India. Methods like Mammography, colonoscopy, CT Scan, and even biopsy and histopathology, and Pap test are beyond the reach of this group, being available only at Multi-Specialty hospitals in big towns and cities. Almost always any type of cancer or cardiovascular diseases are diagnosed only after overt symptoms appear, by which time it is too late for successful therapy. The result of lack of routine screening for early detection is particularly seen in the various cancers, designated as "Number 2 " Killer in almost all countries [19]. But, presumably due to inadequate records only being available in rural areas, it is greatly under-estimated as only the fourth killer in India [17]. Coronary diseases and various types of cancers are the two most important "Killer" diseases where adequate health care can considerably reduce the health care burden of the country. Below we discuss 
how optical biopsy and optical pathology are extremely well tailored at present to meet this need.

One of the major causative factors for cardiovascular diseases is smoking. The use of tobacco (in the form of beedi) and consumption of alcohol (as toddy, desi-spirit, etc.) are relatively high in rural population, while cigarette use and foreign liquor consumption are higher in urban areas [20]. Physical inactivity and obesity, the other major causative factors, may not play a large role for the Indian rural population, which consist mostly of farm laborers, daily wage workers, and middle class people, all of whom have some minimal physical activity in their daily life. Smoking is also recognized as a major causative factor for lung and oral cancers. Optical biopsy and optical pathology can easily recognize quite early, changes induced in tissue and body fluids like saliva and blood by smoking, so that the subjects can be made aware of their health status by periodic observation through screening by optical methods. Fig. 4 shows the fluorescence spectra of oral buccal mucosa for normal, malignant, and smoker subjects. It is seen that in smokers there is a considerable decrease in relative fluorescence intensity in the 375-425 nm region. Excitation of tissue at $325 \mathrm{~nm}$ gives fluorescence in this region from collagen $(386 \mathrm{~nm})$ and pyridoxine-VitaminB $6, \quad(406 \mathrm{~nm})$ derivatives [21]. The decrease in intensity in this region can be attributed to a thickening of the epithelial layer (collagen decrease, $380 \mathrm{~nm}$ ) and loss of Pyridoxine (Vitamin $\mathrm{B}_{6}, 405 \mathrm{~nm}$ ).

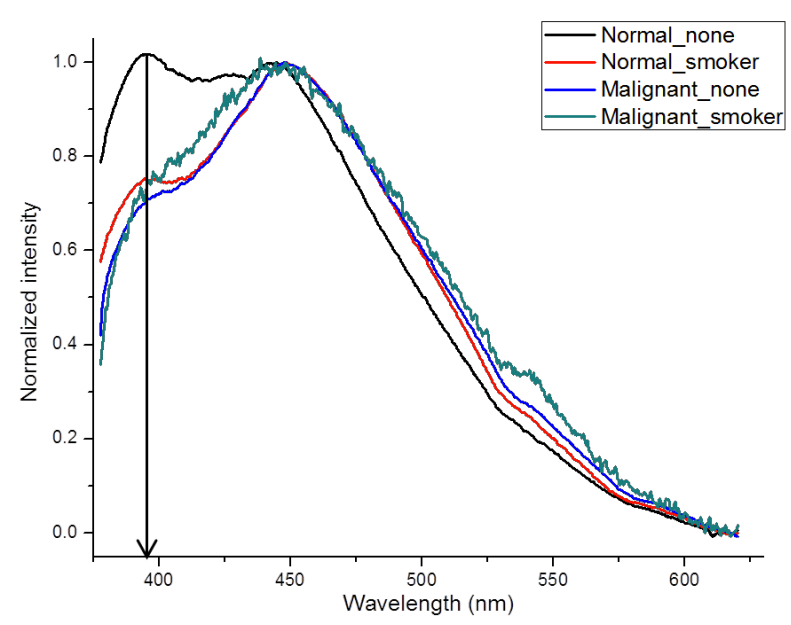

Fig. 4 Buccal Mucosa fluorescence spectra.

Vitamin $\mathrm{B}_{6}$ (Pyridoxine 5'-phosphate) is needed in the production of many neurotransmitters (serotonin, norepinephrine). More important in the present context is the fact, that $\mathrm{B}_{6}$ together with $\mathrm{B}_{12}$ and $\mathrm{B}_{9}$ control the levels of Homocysteine, an amino acid associated with heart diseases, in the blood. A deficiency of $\mathrm{B}_{6}$ in the diet thus poses a higher risk of heart disease [22, 23]. If the decrease in intensity is at least partly due to Pyridoxine, then it is clear that a periodic screening of smokers for signs of loss of pyridoxine intensity can be used to caution them about possible heart problems.
Fig. 4 also shows that the in vivo fluorescence spectra are quite suitable for discrimination between normal and malignant conditions. This is further illustrated in Fig. 5, where the fluorescence differences between normal, pre-malignant, and malignant conditions for buccal mucosa are shown. As mentioned earlier, we have employed PCA and ANN to develop screening and diagnostic methods for early detection of pre-malignant and malignant conditions at the different sites of oral cavity by this method of optical biopsy [8$10,14,16]$. The method of laser induced fluorescence works very well for the detection of malignant conditions of breast and cervical tissues. Similar to oral tissue, excitation at $325 \mathrm{~nm}$ is also quite suitable for the discrimination of disease conditions in breast and cervical tissues based on their fluorescence characteristics (Fig. 6).

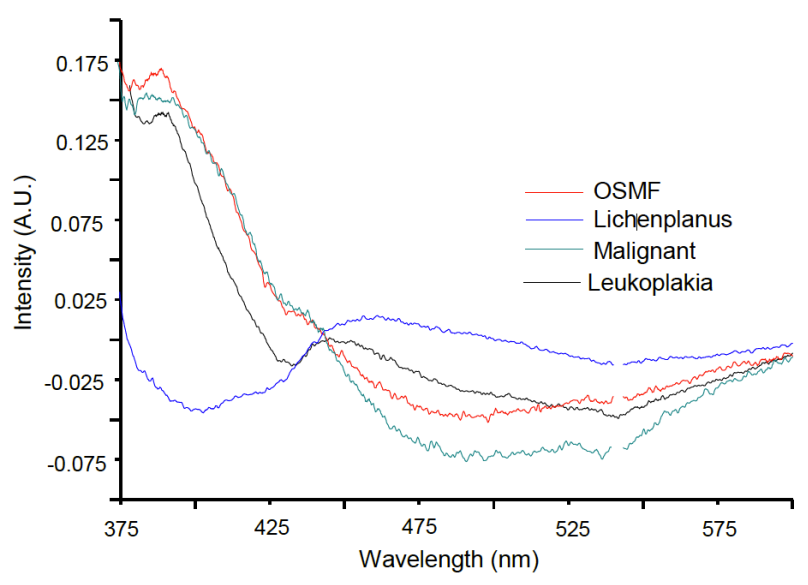

Fig. 5 "Difference Spectra" - Normal Buccal Mucosa (minus) Buccal Mucosa under Different Clinical conditions.

It is appropriate to mention here that, for discriminating between normal and malignant tissue conditions, one can make use of the differences in tissue conditions in the two states. Since the tissue structure, components, and environment of the fluorescing molecular species will be quite different in the two cases, fluorescence, which is highly sensitive to these parameters, will be determined to a very large extent by the intermolecular interactions and environments of the fluorescing molecule. This changes the relaxation processes of the excited molecule, causing drastic changes in excited state lifetimes of the molecular species under investigation. Thus, time resolved fluorescence emission will have different time dependence in the two cases, and may be a very good method for discrimination between normal and malignant or other disease conditions. This is illustrated in Fig. 7 in the case of breast tissue. It is seen that the fluorescence at $400 \mathrm{~nm}$, arising from Pyridoxine/Collagen, decay very fast in malignant condition, whereas it shows an initial increase and a very slow decay rate afterwards, for normal tissue.

It is well recognized that a host of biochemical reactions precede/accompany the onset of any disease. 

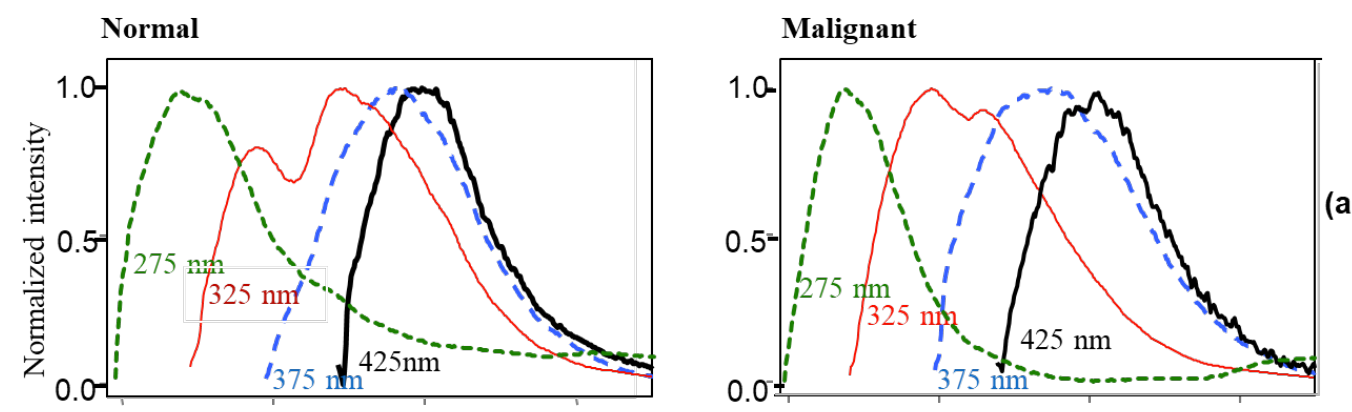

(a)
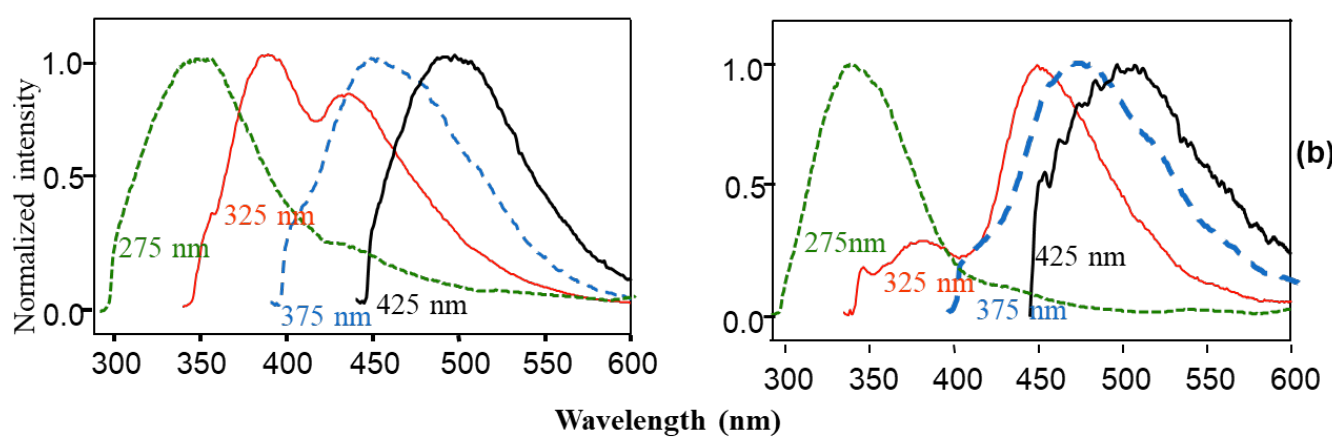

Fig. 6 Fluorescence spectra of normal and malignant breast (a) and cervical tissues (b) at different excitation wavelength.

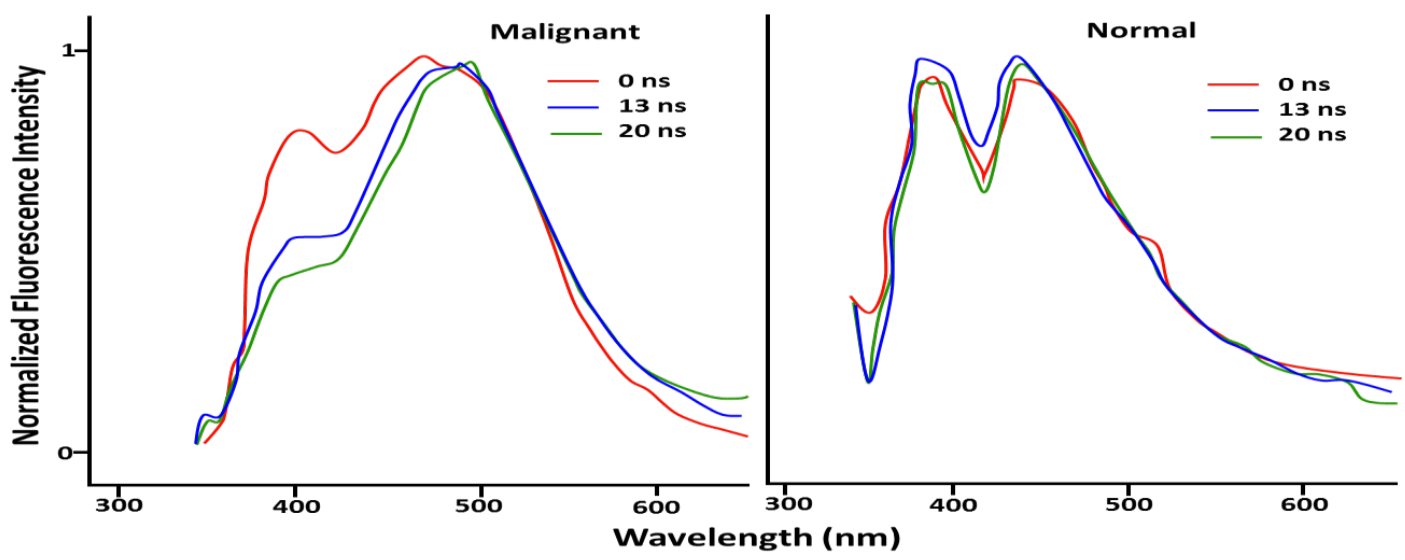

Fig. 7 Breast tissue; Time dependence of fluorescence: excitation $325 \mathrm{~nm}$.

Changes continue to take place during progression of the disease, and also in regression during therapy. These changes will be reflected in the cellular components, and circulating body fluids, as in the case of tissue sites. We have shown that protein profiling of body fluids, cytological specimens, and biopsy tissue samples provide unambiguous markers, which can discriminate between normal and disease conditions. They can be used for screening, early detection, staging, and followup for deciding efficacy of therapy. Having seen a direct connection between smoking and coronary diseases (and oral cancer), and tissue fluorescence, it should be interesting to look at protein profiles of body fluids under similar situations. We have developed an ultrasensitive protein profiling system (Fig. 8) based on highly efficient High Performance Liquid Chromatograpy-Ultrasensitive Laser Induced Fluorescence (HPLC-LIF) for this. The details of the system and its extensive use in monitoring various cancers (oral, cervical, ovarian, breast, urinary bladder, colon, etc.), coronary conditions, gynecological problems, etc. are described elsewhere [24-30]. Here we will illustrate the capabilities of the system with a few results.

In view of the changes observed in oral tissue (Fig. 4), it will be informative to see whether similar striking changes happen in the tissue homogenates, body fluids in potentially malignant and malignant conditions. Figs. 9 and 10 show protein profiles recorded using the HPLC-LIF system (separation column was reverse phase biphenyl and mobile phase: water/acetonitrile/TFA), of oral tissue homogenates (normal and malignant), and saliva under normal, premalignant, and malignant conditions to different sites in the oral cavity. 


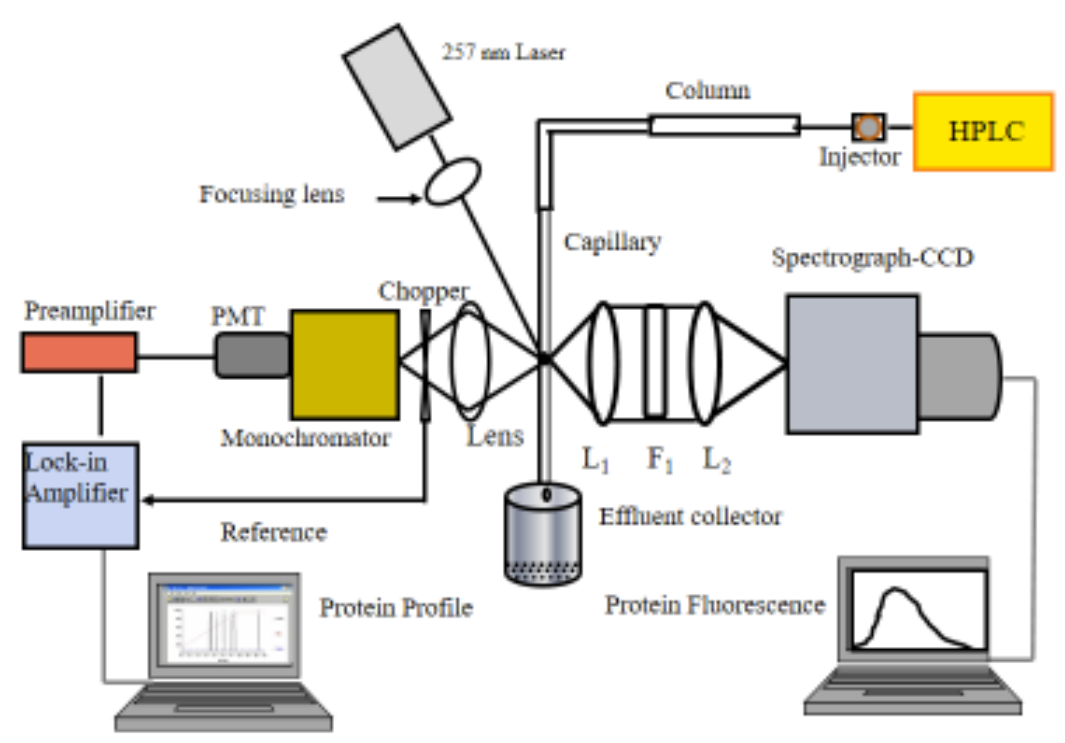

Fig. 8 Block diagram of the locally assembled HPLC-LIF system.

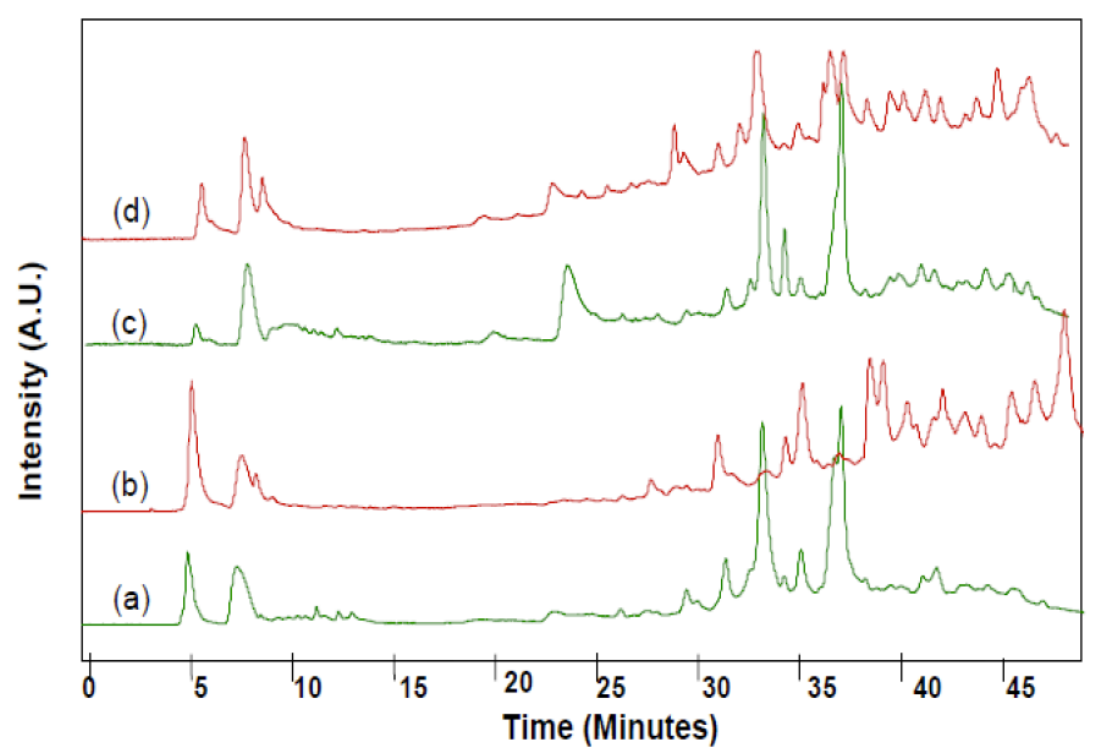

Fig. 9 Protein profiles oral tissue homogenates of (a) Male normal (55 Years); (b) Male malignant (55 Years); (c) Male normal (62 years) and (d) Male malignant (62 years).

It is seen that there are substantial changes from the normal saliva under all conditions, showing that drastic changes have taken place in the functioning of the associated organs. We have standardized the protein profile pattern analysis method for screening, early detection, and monitoring of oral cancer by PCA of salivary samples, with high sensitivity and specificity [24].

Blood, which circulates all over the body, collects many bio-molecules produced in different organs, and also serves as a distributor for molecular species like anti-bodies, antigens, immunoreaction products and many other molecules. We have investigated the protein profile of blood in many different conditions, normal, smoker, various malignancies, cardiovascular diseases, diabetes, etc. Fig. 11 shows typical chromatograms of serum from normal, smoker, oral premalignant and malignant subjects. 


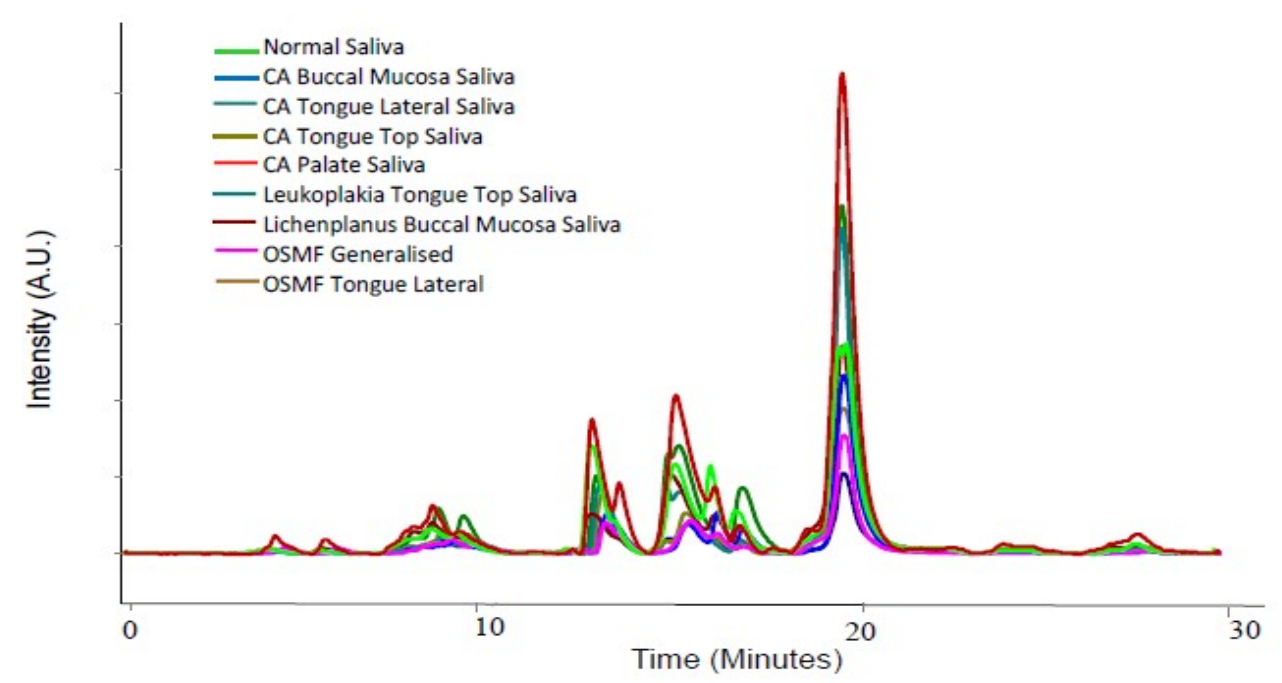

Fig. 10 Protein profiles human saliva: Normal saliva, Cancer of buccal mucosa, cancer of tongue lateral, cancer of tongue top; cancer of palate and some other potentially malignant cases.

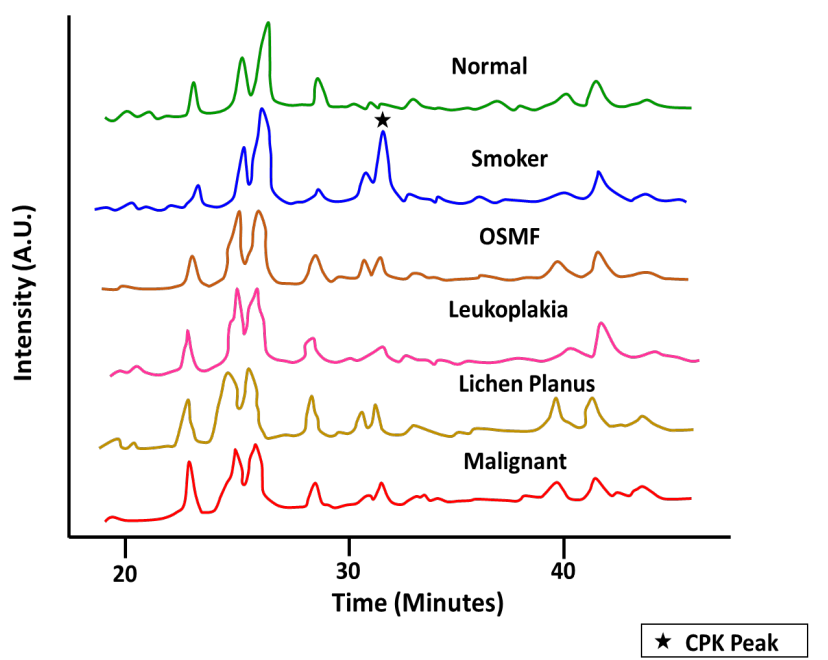

Fig. 11 HPLC-LIF Protein profiles of serum samples.

We have identified many of the peaks here, including transferrin, ferritin, $\mathrm{HSA}, \mathrm{I}_{\mathrm{G}} \mathrm{G}$, and $\mathrm{CPK}$, by co-injection of pure compounds as well as SDS Page of the collected fractions. PCA of the serum protein profiles have been shown to be highly suitable for screening, early detection, staging, and follow-up in therapy [27-30] for many cancers and other abnormal conditions.

It is seen from Fig. 11 that CPK (peak $\sim 32 \mathrm{~min}$ ), which is present in extremely small amounts in normal serum - our detection limit for CPK is about $7 \mathrm{fmol}$ [31] - increases to very large values in smokers, and is also high in pre-malignant and malignant conditions of the oral cavity. Fig. 12 shows a plot of relative intensities of CPK peaks in a number of samples with reference to a standard peak, which had remained more or less same in all samples.

The levels of CPK, together with other proteins like
Troponin I (TnI) and Troponin $\mathrm{T}(\mathrm{TnT})$ are linked with injury of the heart muscle [32-35]. An injury of heart muscle like that from a heart attack, leads to an increase in their levels in the bloodstream. This is supported by protein profile study of serum samples after ischemic heart disease, shown in Fig. 13. The observation of CPK levels in serum of smokers can thus provide a method for screening and diagnosis of coronary problems.

The change in the relative intensities of HSA and CPK illustrate the "catastrophic" change in CPK in Ischemic Heart Disease.

In many cancers often biopsy samples are obtained by procedures like brush biopsy, fine needle aspiration, or by mechanical removal of cellular samples. These cytological samples are then processed and examined by a pathologist, under a microscope. Diagnosis and staging of the disease is arrived at by visual observation of morphological or biomolecular changes. Thus, the results are subjective to the experience of the pathologist, and are prone to other problems like "Fatigue Factor" arising from need to observe large number of samples over long periods. Also, during early stages of the disease, the number of cells in abnormal condition may be quite small and may be missed leading to "False Negative" results. On the other hand, often changes may take place due to reasons other than the suspected disease, and this may lead to "False positive" diagnosis. Such problems can be minimized by optical pathologic techniques like "Spectral Histopathology" and "Spectral cytopathology", and ultrasensitive HPLC-protein profiling, which can detect presence of even extremely small number of abnormal cells in a collection with very large number of normal cells. We have standardized such protein profiling method of cellular samples, for, screening, early diagnosis, staging and follow-up in therapy [26, 36]. Especially noteworthy is the fact that the protein profile 


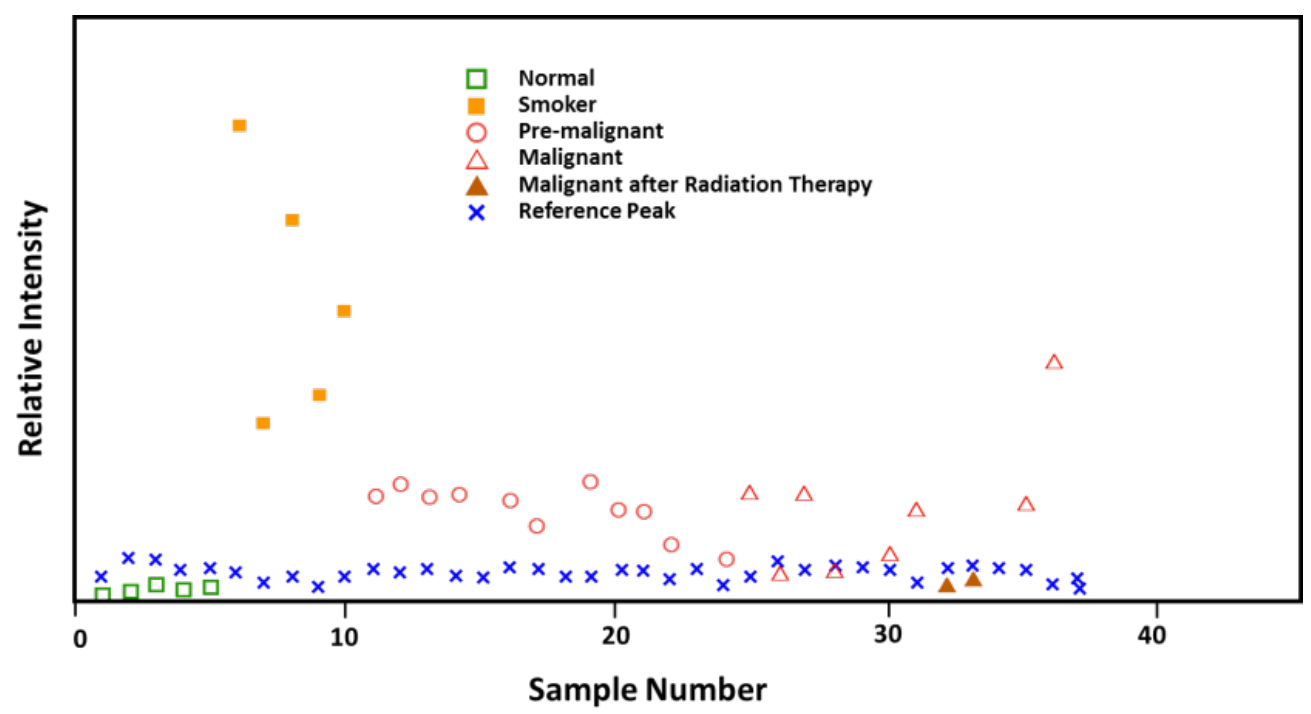

Fig. 12. Relative Intensity of CPK in Serum samples of different conditions.

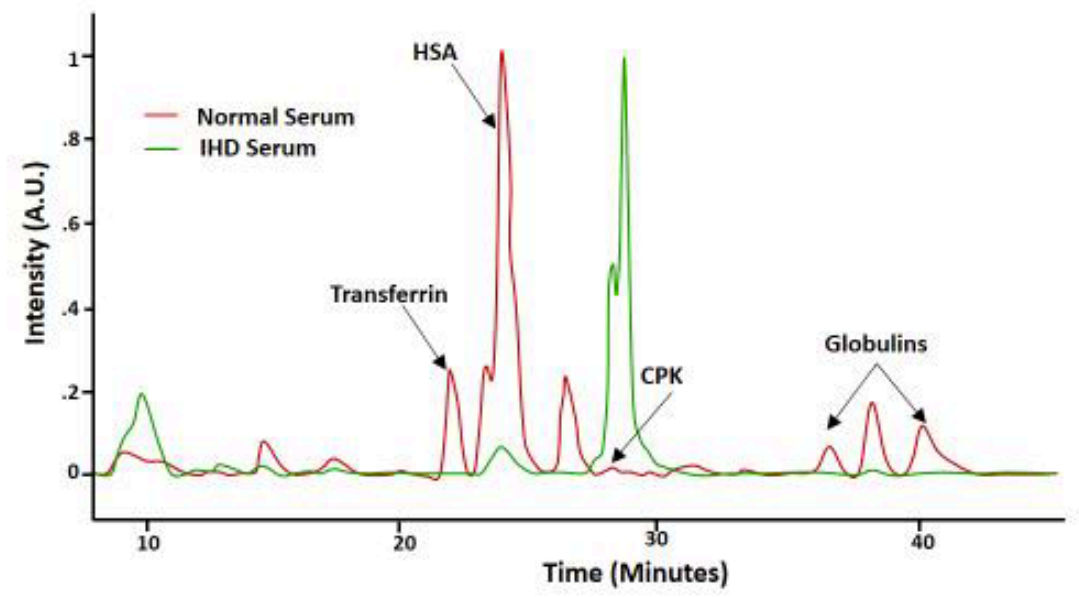

Fig. 13 HPLC-LIF Protein Profiles of Serum in Ischemic Heart Disease.

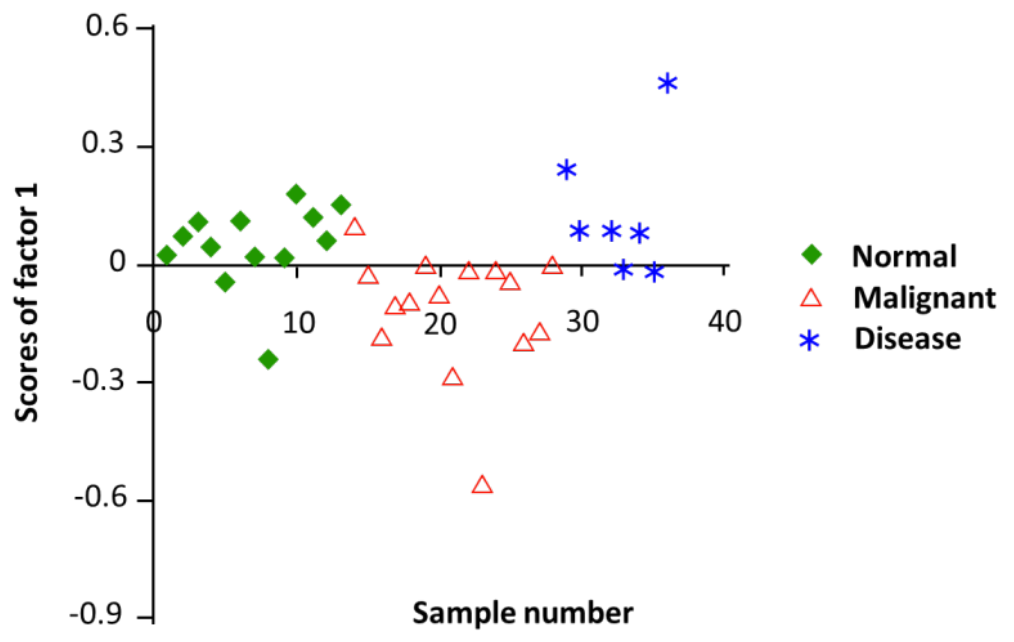

Fig. 14 PCA Scores for factor 1: Cytological Samples from Cervix. 
study could discriminate other disease conditions from malignancy, as shown in Figure 14, where the scores of Factor 1 from PCA of serum protein profiles are plotted against sample number. It is seen that subjects having diseases of cervix other than cervical cancer (erosion of cervix, nabothian cyst, polycystic ovary, etc.), are separated out from subjects with cervical cancer.

\section{Conclusion}

It is clear from the results presented above and the various work referred to in the text, that advances in optical instrumentation, data processing techniques, and development of efficient analytical methods based on ultrasensitive spectroscopy techniques, have made Optical Biopsy and Optical Pathology acceptable and affordable for the majority-rural population in developing countries, satisfying the requirements of many health care needs like screening, early detection, diagnosis, and therapy follow-up in many diseaseconditions. The techniques can be made universally available in resource limited settings in developing countries, especially because of advantages like costeffective instrumentation, which can be made easily portable, and which can be operated by trained technicians without the need for highly qualified medical professionals like clinicians, radiologists, oncologists, and pathologists. Also diagnostic decisionmaking being done with objective statistical methods, minimizing possible human errors like false positive and false-negative conclusions, over-diagnosis (as in most imaging methods), and errors that can arise from multiple choices. Methods like serum protein profile are minimally (personally) invasive making it acceptable to all levels of population. Also, collection, storage, transportation, processing etc. for blood samples are highly standardized and, thus, it is quite convenient for large scale screening operations. Thus, it is highly desirable to encourage the acceptance of Optical Biopsy and Optical Pathology techniques as routine technology to provide affordable health care to the $70 \%$ of the rural population in developing countries like India, who at present survive with grossly inadequate health care facilities. It should also be emphasized that such rural health care can in effect provide much better successful therapy by the multispecialty hospitals in big towns and cities, since they get the opportunity to start treatment at early stages, of suspect-subjects, screened and referred to them by the local hospitals, clinics and health care units.

\section{Disclosures}

The authors declare that there are no conflicts of interest related to this article.

\section{Acknowledgement}

Authors are thankful to VGST, Govt. of Karnataka, BRNS, DST, DBT, Govt. of India and Manipal Academy of Higher Education for financial support for the Optical Pathology research initiatives. Swathi Rao and Reena John are thankful to Dr. TMA Pai Ph.D. Scholarship and Govt. of Karnataka Minority Scholarship respectively.

\section{References}

1. “World Population Prospects: The 2019 Revision," United Nations Population Division (accessed April 4, 2020).

2. OECD Health Statistics 2020 (accessed May 17, 2020).

3. GBD 2015 SDG Collaborators, "Measuring the health-related Sustainable Development Goals in 188 countries: a baseline analysis from the Global Burden of Disease Study 2015," The Lancet 388(10053), 1813-1850 (2016).

4. V. B. Kartha, Santhosh C., Biomedical Spectroscopy, (Chapter 1, and References therein), Manipal University Press, Manipal, India (2014).

5. A. F. Fercher, W. Drexler, C. K. Hitzenberger, and T. Lasser, “Optical coherence tomography - principles and applications," Reports on Progress in Physics 66, 239-303 (2003).

6. S. L. Jacques, "Optical properties of biological tissues: a review," Physics in Medicine and Biology 58(11), R37R61 (2013).

7. V. V. Tuchin, "Light scattering study of tissues," Physics Uspekhi 40(5), 495-515 (1997).

8. B. K. Manjunath, J. Kurien, L. Rao, C. Murali Krishna, M. S. Chidananda, K. Venkatakrishna, and V. B. Kartha, "Autofluorescence of oral tissue for optical pathology in oral malignancy," Journal of Photochemistry and Photobiology B: Biology 73(1-2), 49-58 (2004).

9. S. M. Chidananda, K. Satyamoorthy, L. Rai, A. P. Manjunath, and V. B. Kartha, "Optical diagnosis of cervical cancer by fluorescence spectroscopy technique,” International Journal of Cancer 119(1), 139-145 (2006).

10. V. B. Kartha, J. Kurien, K. M. Pai, L. Rao, L. Rai, K. K. Mahato, C. M. Krishna, and C. Santhosh, "Diagnosis at the molecular level: Analytical Laser spectroscopy for clinical applications," Chapter in Photo/Electrochemistry \& Photobiology in the Environment, Energy and Fuel, S. Kaneco (Ed.), Research Signpost, Trivandrum, 153-221 (2005).

11. R. Jyothi Lakshmi, M. Alexander, J. Kurien, J. G. R. Solomon, P. Umadevi, M. S. Vidyasagar, and V.B. Kartha, "Laser spectroscopy studies of radiation effects on animal models and human systems," Chapter in 
Photo/Electrochemistry \& Photobiology in the Environment, Energy and Fuel, S. Kaneco (Ed.), Research Signpost, Trivandrum, 105-151 (2005).

12. Biopsy: Types of biopsy procedures used to diagnose cancer, Mayo Clinic Staff (accessed 16/07/2016).

13. P. Holmstrup, P. Vedofte, J. Reibel, and K. Stoltze, “Oral pre-malignant lesions: is a biopsy reliable?" Journal of Oral Pathology \& Medicine 36(5), 262-266 (2007).

14. A. Patil, V. K. Unnikrishnan, R. Ongole, K. M. Pai, V. B. Kartha, and S. Chidangil, "Non-Invasive in vivo Screening of Oral Malignancy," Modern Technologies in Medicine 10(1), 15-26 (2018)

15. V. K. Unnikrishnan, A. Patil, R. Bernard, K. K. Mahato, K. M. Pai, R. Ongoleetal, "Oral Cancer Screening in Rural India-Report on in vivo Device Test of 349 Subjects at Manipal and Mangalore," Philips Research, Technical Note MU-PR-TN/LIF-02/2007 (2007).

16. C. E. Metz, "Basic Principles of ROC Analysis," Seminars in Nuclear Medicine 8(4), 283-298 (1978).

17. What's Killing India? Knowing Top 10 Killer Death Diseases in India, icicilombard.com (accessed Aug 06, 2013).

18. A. Jemal, F. Bray, M. M. Center, J. Ferlay, E. Ward, D. Forman, “Global Cancer Statistics," CA: A Cancer Journal for Clinicians 61, 69-90 (2011).

19. Global Cancer Facts and Figures, 3rd Edition, American Cancer Society, Atlanta, Georgia (2015).

20. Household Consumption of Various Goods and Services in India, 2011-12, NSS Report No. 558, Ministry of Statistics and Programme Implementation, Government of India, June (2014).

21. N. Ramanujam, "Fluorescence spectroscopy In Vivo," Chapter in Encyclopedia of Analytical Chemistry, R.A. Meyers (Ed.), John Wiley \& Sons, 20-56 (2000).

22. Vitamin B6 (Pyridoxine), University of Maryland Medical Centre (accessed 8/5/2015).

23. H. McNulty, K. Pentieva, L. Hoey, and M. Ward, "Homocysteine, B-vitamins and CVD," Proceedings of the Nutritions Society 67(2), 232-237 (2008).

24. A. K. Patil, K. S. Choudhari, V. K. Unnikrishna, N. Shenoy, R. Ongole, K. M. Pai, V. B. Kartha, and C. Santhosh, "Salivary protein markers: a noninvasive protein profile-based method for the early diagnosis of oral premalignancy and malignancy," Journal of Biomedical Optics 18(10), 101317 (2013).

25. S. Bhat, A. Patil, L. Rai, V. B. Kartha, and S. Chidangil, "Application of HPLC Combined with Laser Induced Fluorescence for Protein Profile Analysis of Tissue Homogenates in Cervical Cancer," The Scientific World Journal 2012, 976421 (2012).

26. S. Bhat, A. Patil, L. Rai, V. B. Kartha, and C. Santhosh, "Protein profile analysis of cellular samples from the cervix for the objective diagnosis of cervical cancer using HPLC-LIF," Journal of Chromatography B 878(31), 3225-3230 (2010).

27. A. Patil, V. Prabhu, K. S. Choudhari, V. K. Unnikrishnan, S. D. George, R. Ongole, K. M. Pai, K. Jayarama Shetty, S. Bhat, V. B. Kartha, and C. Santhosh, "Evaluation of high-performance liquid chromatography laserinducedfluorescence for serum protein profiling for early diagnosis of oral cancer", Journal of Biomedical Optics, 15(6), 067007 (2010).

28. L. Rai, P. Kumar, K. K. Mahato, V. B. Kartha, and C. Santhosh, "Serum protein profile study of normal and cervical cancer subjects by high performance liquid chromatography with laser-induced fluorescence," Journal of Biomedical Optics 13(5), 054062 (2008).

29. S. K. Singh, R. L. Martis, S. Bhat, A. Rani, P. Kushtagi, L. Rai, V. B. Kartha, and C. Santhosh, "Protein Profile Study of Clinical Samples of Ovarian Cancer using High Performance Liquid Chromatography-Laser Induced Fluorescence (HPLC-LIF)," Proceedings of SPIE 6092, 60920N (2006).

30. V. K .Venkatakrishna, B. Kartha, K. M. Pai, C. M. Krishna, O. Ravikiran, J. Kurian, M. Alexander, and G. Ullas, "HPLC-LIF for Early Detection of Oral Cancer," Current Science 84(4), 551-557 (2003).

31. A. Patil, K. S. Choudhari, V. Prabhu, V. K. Unnikrishnan, S. Bhat, K. M. Pai, V. B. Kartha, and C. Santhosh, "Highly Sensitive High Performance Liquid Chromatography-Laser Induced Fluorescence for Proteomics Applications,” International Scholarly Research Notices 2012, 643979 (2012).

32. J. F. Portales, J. A. García Robles, J. J. Candil, E. P. David, J. R. Rey Blas, L. Pérez de Isla, Ó. D. Castro, and J. Almendral, "Utility of the Serum Biochemical Markers CPK, CPK MB Mass, Myoglobin, and Cardiac Troponin T in a Chest Pain Unit. Which Marker Determinations Should Be Requested and When?" Revista Española de Cardiología 55(9), 913-920 (2002).

33. Cardiac Enzyme Studies:Test Overview WebMD Medical Reference from Healthwise (acessed August 7, 2015).

34. F. Fischbach, D. M. Bunning III, A Manual of Laboratory and Diagnostic Tests, 9th ed., Wolters Kluwer Health, Philadelphia (2015).

35. N. Sujatha, L. Rai, P. Kumar, B. R. Krishnanand, K. K. Mahato, S. D. George, V. B. Kartha, S. C, "Protein profile study of Pap smear and tissue of cervix by High Performance Liquid Chromatography-Laser Induced Fluorescence," Proceedings of SPIE 6441, 64410B (2007). 